\title{
A LOW-VOLTAGE KLYSTRON FOR THE ILC AND ILC TESTING PROGRAM*
}

\author{
N. Barov", N. Moshman, L. Zhao, J.S. Kim, FAR-TECH, Inc., San Diego, CA 92121, USA
}

\section{Abstract}

FAR-TECH, Inc. is developing and building a $36 \mathrm{kV}$, $830 \mathrm{~kW}$ klystron for the International Linear Collider (ILC) testing program. A variant of the tube can also be used to supply RF energy for a 2 or 3 meter section of ILC. The tube design is of the multiple-beam klystron (MBK) type, using ten beams with confined flow focusing. The design optimizes small tube size and low cost. The initial prototype will use an electromagnet, but the design allows for the eventual use of a permanent magnet solenoid. An efficiency of $65 \%$ is expected. We will present the design and status of the construction of the klystron and supporting systems.

\section{INTRODUCTION}

The baseline International Linear Collider (ILC) RF system design has a collection of $10 \mathrm{MW}$ klystrons and modulators, and a distribution system that directs the RF power to each cavity input coupler. In addition to the multiple beam klystrons (MBK) developed by Thales[1], CPI[2], and Toshiba[3], several alternative klystron designs have also been proposed. SLAC has been working on a sheet-beam klystron design, as well as an alternative PPM-focused round-beam klystron[3]. KEK has proposed a low-voltage, $10 \mathrm{MW}$ klystron[5] that aims to simplify the modulator design.

FAR-TECH, Inc. has been developing a klystron that operates at reduced power $(830 \mathrm{~kW}, 1 / 12$ of a standard ILC klystron). Initially this klystron will be used in the ILC testing program at FNAL. This tube design also offers several advantages as an ILC power source. The low-voltage design $(36 \mathrm{kV})$ can simplify the modulator and klystron gun socket design. The RF distribution system can be simplified and its losses reduced. During operation, the linac would more easily tolerate the loss of a single klystron, and the RF feedback system would be simplified.

A detailed cost analysis for large-scale production of such a tube has not been carried out. However, the design so far has emphasized a cavity geometry that is simple to produce, as is later discussed. Also, as an alternative to the $10 \mathrm{MW}$ designs, the tube would be produced in far larger numbers, around 8000 for ILC, and therefore sets a different economy of scale in terms of the eventual unit cost.

\section{KLYSTRON PARAMETERS}

The first prototype will be constructed using a constant guide magnetic field, with no beam convergence after emission. Subsequent tubes can be built with the convergent gun design. Both gun designs are discussed in the following section. The parameters of the first prototype are summarized in Table 1, and the initial prototype will resemble the image shown in Figure 1.

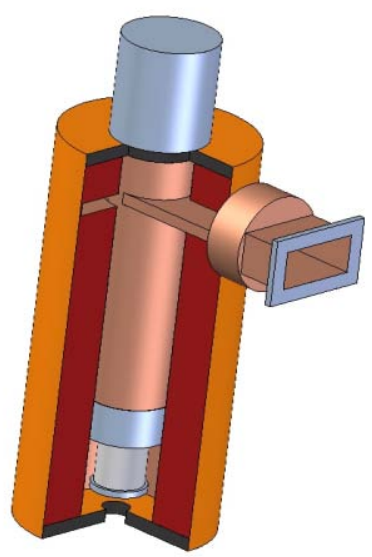

Figure 1: The klystron design envisioned for the first prototype.

Table 1: Klystron Parameters

\begin{tabular}{|l|c|}
\hline Parameter & Value \\
\hline Operating frequency $(\mathrm{MHz})$ & 1300 \\
\hline Bandwidth, -1 dB (MHz) & 5.3 \\
\hline Saturation gain $(\mathrm{dB})$ & 55 \\
\hline Number of beams & 10 \\
\hline Saturated power $(\mathrm{kW})$ & 830 \\
\hline Efficiency (percent) & 65 \\
\hline Magnetic guide field (Gauss) & 1400 \\
\hline Cathode loading (A/cm $\left.{ }^{2}\right)$ & 5.2 \\
\hline No. cavities (fundamental) & 5 \\
\hline No. cavities (2nd harmonic) & 1 \\
\hline RF pulse length (ms) & 1.6 \\
\hline RF repetition frequency (Hz) & 5 \\
\hline Beam voltage (kV) & 36 \\
\hline Beam current (Amps) & $10 \times 3.4$ \\
\hline
\end{tabular}

\section{GUN DESIGN}

\section{Convergent gun design}

In this design, the cathode loading is kept small $(<2$ $\mathrm{A} / \mathrm{cm}^{2}$ ) which ensures a long tube life. A micro-perveance
*Work supported by US Dept. of Energy.

"barov@far-tech.com 
of 0.5 for each of the ten 3.4 A beams allows for highefficiency operation of the klystron. The beam radius is manipulated by a magnetic field that is ramped up from the value of $\sim 130$ Gauss on the cathode to $\sim 500$ Gauss in the intermediate region, and then to the full 800 Gauss in the gain-cavity section of the tube. Two iron plates in the body of the tube are used to modify the longitudinal field profile. Figure 2 Shows an EGUN simulation of this gun. With proper choice of the magnetic field profile and length between the two plates, it is possible to achieve a perfectly matched beam envelope into the downstream region of the tube. This design would benefit from fully 3-D gun and magnetic field simulations, which have not been performed at this stage. No fundamental problems, however, are anticipated in the fully 3-D case.

In both this and the non-convergent design, the beams are offset by $4.2 \mathrm{~cm}$ from the axis of the tube.

\section{Non-convergent gun design.}

For the first prototype, we are adopting a nonconvergent gun design. In order to excessive scalloping, the magnetic field is raised to 1400 Gauss, which results in a scalloping of $\sim 10 \%$. The EGUN-generated beam distribution was transferred directly into a 2-D MAGIC simulation, with no loss of tube efficiency vs. the perfectly matched case.

In this case, the beams are far enough apart that any corrections from a fully 3-D treatment of the gun system are likely to be negligible.

The solenoidal field is set up by an electromagnet that covers the gun and gun socket as well as the gain cavity section (see Figure 1). A POISSON model of the solenoid is shown in Figure 3. An iron plate terminates the magnetic field at the collector entry. The solenoid coil also needs to be interrupted in the region of the output waveguide. Without this waveguide slot, the transverse magnetic field can be kept below 1 Gauss (assuming a perfectly constructed solenoid). With the slot, and suitable correction with a set of trimmer coils, the transverse field seen by the beams is kept below 7 Gauss, or $0.5 \%$ of the longitudinal field.

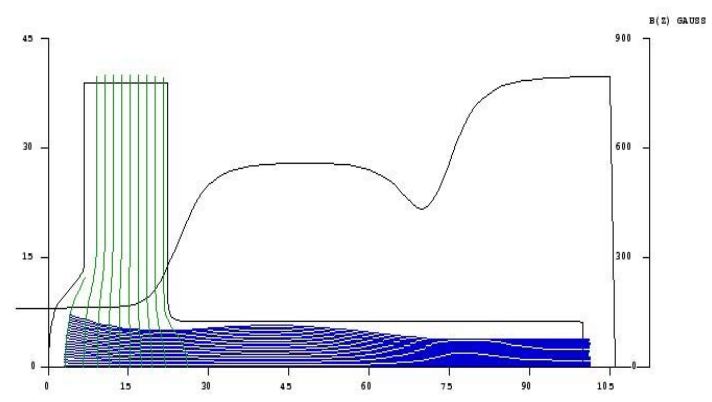

Figure 2: EGUN simulation of the convergent gun design.

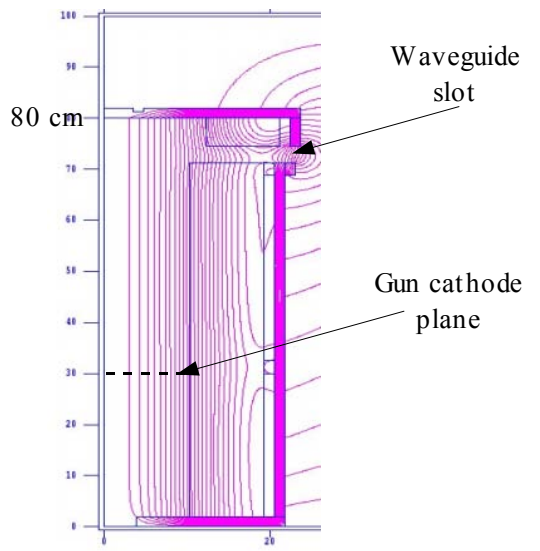

Figure 3: A 2-D model of the soleonid magnetic field profile, including the main coil, top coil, and two trimmer coils.

\section{GAIN CAVITIES}

The tube contains five cavities operating near the fundamental frequency, and one (the fourth cavity) at the second harmonic. All cavities are common to the ten beams, and are of the ring-resonator design. The entire gain cavity section fits within a volume of $15 \mathrm{~cm}$ diameter by $50 \mathrm{~cm}$ length. The overall low volume simplifies production of the cavities for this tube design.

The gain cavities were simulated with HFSS, and the entire beam-cavity system was extensively simulated with MAGIC 2-D to arrive at a case with optimized efficiency.

Figure 4 shows the result of bandwidth calculations performed with MAGIC 2-D. These results were performed with the input cavity having an artificially wide bandwidth, which results in the dip at the center frequency and the overall slope of flat-top with less gain at higher frequencies. Using the correct input cavity model is expected to correct these features.

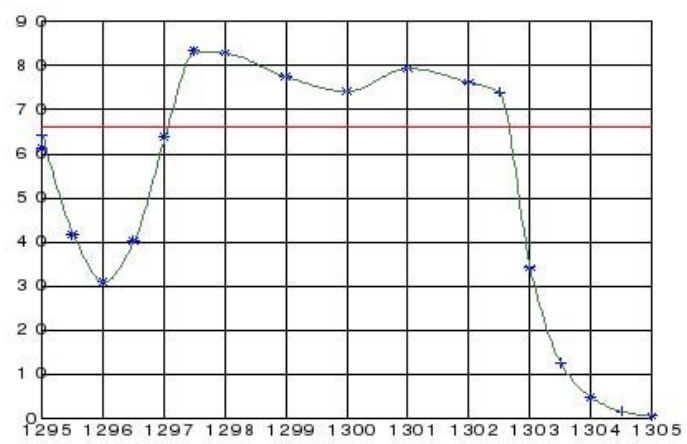

Figure 4: Bandwidth calculation using MAGIC. The horizonal axis is frequency in $\mathrm{MHz}$, and the vertical axis is power per beam in $\mathrm{kW}$.

Figure 5 shows the geometry of the copper pieces for producing the cavities. The geometry shown includes the volume of two adjacent half-cavities. The entire gain cavity section will be constructed by stacking together a number of such pieces.

We are continuing efforts to perform fully 3-D simulations of the klystron performance. However, these 
simulations are more difficult to set up, and are much more demanding in terms of computing resources. We have begun setting up computational models using MAGIC 3-D, and LSP. These studies will allow us to evaluate the impact of unequal currents for each of the ten beams, or a complete failure of one of the cathodes.

A challenge with these codes is how to bypass the first $1 \mu \mathrm{s}$ of the simulation, during which the tube has not yet reached steady-state operation. We will address this with another approach involving a new code being developed at FAR-TECH called PARALACS. Like the other codes mentioned above, this code uses an explicit push of the Maxwell equations, and a charge-conserving particle advance. The code is being developed in C, and uses MPI for parallel communications.

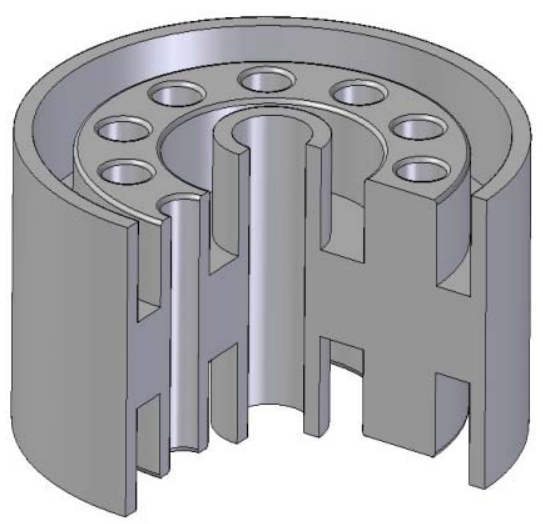

Figure 5: Geometry of copper piece that includes the endwalls of two adjacent ring cavities.

\section{COLLECTOR AND OUTPUT WINDOW}

The collector is envisioned to be common to all 10 beams. Simulations indicate no problems with secondary emission or back-scattered primary electrons for a collector having a $25 \mathrm{~cm}$ length.

For the initial prototype, the $<1 \mathrm{MW}$ output power can be handled with an off-the-shelf window design.

\section{CONCLUSION}

Having completed the majority of the simulations and design for this tube, construction and testing will be carried out during the following year.

\section{REFERENCES}

[1] A. Beunas, G. Faillon, S. Choroba, "A High Power Long Pulse High Efficiency Multi Beam Klystron", $5^{\text {th }}$ Modulator-Klystron Workshop for Future Linear Colliders, (2001).

[2] A. Balkcum, et al., "Design and Operation of a HighPower L-Band Multiple Beam Klystron”, 2005 IEEE Particle Accelerator Conference.

[3] Y.H. Chin, A. Yano, S. Mikage, S. Choroba, "Development of Toshiba L-Band Multiple Beam Klystron for European XFEL Project," 2005 IEEE Particle Accelerator Conference.

[4] D. Sprehn, "Klystron Alternatives for the ILC", First ILC Workshop, KEK Tsukuba, Ibaki, Japan Nov. 2004.

[5] T.Shidara, S. Fukuda, "Alternative Low Voltage Power Source," ALCPG \& ILC Workshop, Snowmass, CO, (2005).

[6] MAGIC User's manual, ATK, Inc., Mission Systems Group. 